\title{
HST Observations of PSR J2051-0827: Asymmetry, Variability and Modelling
}

\author{
B. W. Stappers \\ University of Amsterdam, Kruislaan 403,1098 SJ Amsterdam, NL
}

M. van Kerkwijk

University of Utrecht, P.O. Box 80000, 3508 TA Utrecht, NL

\section{J. F. Bell}

ATNF, CSIRO, P.O. Box 76, Epping, NSW 1710, Australia

\begin{abstract}
Using the Hubble Space Telescope we have successfully detected the "dark" side of the optical companion to the eclipsing millisecond pulsar J2051-0827. These data were obtained over a number of system orbits and clearly show that there is variability in the optical flux, particularly at orbital phases after maximum light. Comparison with phases before maximum light suggests this variation is due to an additional component. The data are modelled by a gravitationally-distorted low-mass secondary star which is irradiated by the impinging pulsar wind. The quality of the model fits are however limited by the variability.
\end{abstract}

\section{Introduction}

PSR J2051-0827 is one of only two known eclipsing binary millisecond pulsars with optically identified companions (Stappers, Bessell, \& Bailes 1996a). It has a spin period of $4.5 \mathrm{~ms}$ and an orbital period of just 0.1 days. There is clear evidence for material extending beyond the Roche lobe of the companion and it is likely that this is being lost from the system. Radio timing studies yield a lower limit on the companion mass of $0.025 \mathrm{M}_{\odot}$ (Stappers et al. 1996b), but no information on its radius relative to its Roche lobe. Optical observations of the companion provide the best hope of resolving this question. They can also help determine the nature of the ablation process and may constrain the composition of this extremely light companion. These factors are vital in our understanding of the past and future evolution of the system.

The light curve of PSR J2051-0827 has been studied previously (Stappers et al. 1996a; Stappers et al. 1999) and has been shown to be similar to that of PSR B1957+20 (Callanan, van Paradijs, \& Rengelink 1995). In both cases the companion was modelled by a gravitationally distorted star, irradiated by the pulsar wind. The results for PSR J2051-0827 indicated that more than $30 \%$ of the energy impinging from the pulsar wind is re-radiated as optical emission and that the companion does not fill its Roche lobe. This contrasts 


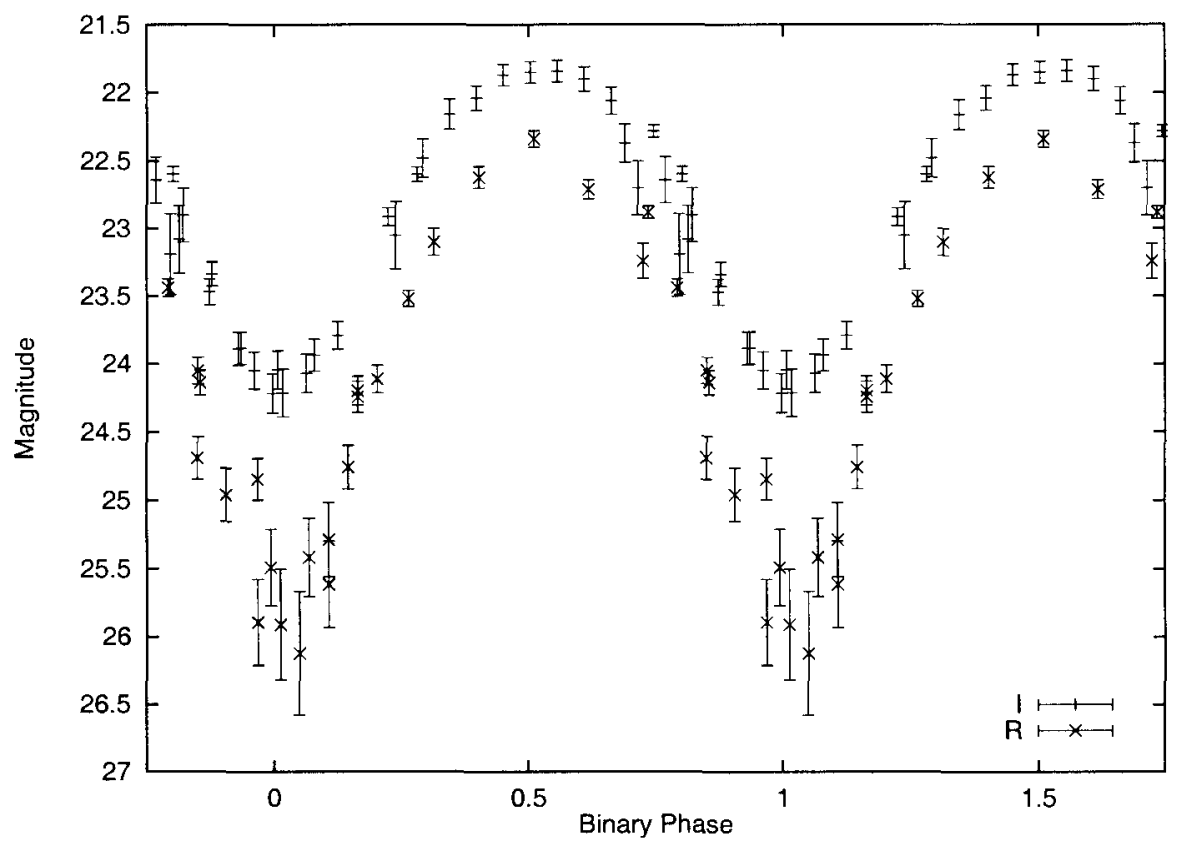

Figure 1. The phased and calibrated Cousins R \& I lightcurve combining observations from the AAT, Palomar and HST. The R magnitude varies by 3.3 magnitudes between the heated and cool side while the I magnitude varies by just 1.9 magnitudes.

with the model for PSR B1957+20 (Callanan et al. 1995) which requires a lower efficiency and a Roche lobe filling companion. This model for PSR J2051-0827 provides evidence that (at present in this system) the pulsar wind is the cause of ablation, rather than tidal dissipation. A limitation of this study is that it was only possible to observe the side of the companion irradiated by the pulsar. The best constraints on the stellar radius and therefore on the the geometry of the system and ablation models would come from studying the complete lightcurve and in particular the cold unirradiated side.

\section{Observations and Modelling}

The Hubble Space Telescope (HST) Wide Field Planetary Camera (WFPC2) was used to obtain a total of 35 images of the PSR J2051-0827 field, spread over 12 orbits of the system, concentrated on optical minimum, in the $6675 \mathrm{w}$ and $8814 \mathrm{w}$ filters. Exposure times ranged between 400 and 500 seconds. Data reduction was carried out in the IRAF software package starting from the pipeline-calibrated PC images. Other observations from Palomar and the AAT that are also used in the following analysis have been described in detail by Stappers et al. (1999).

The pulsar was detected at optical minima in both filters with $\mathrm{m}_{\mathrm{f} 675 \mathrm{w}}=$ 25.9 and $\mathrm{m}_{\mathrm{f} 814 \mathrm{w}}=24.2$. These $H S T$ based magnitudes were then converted to 
the Cousins R \& I based system using the prescription of Holtzman et al. (1995) and then combined with other observations from the literature. Thus a phased and calibrated lightcurve containing 53 observations with good orbital phase coverage was obtained and is shown in Figure 1.

These data were then fitted by a model of an irradiated companion star which included, amongst other things, the affects of gravitational and rotational distortion and gravity darkening. As the unirradiated side of the companion star is likely very cool, the emergent spectrum needs to be modelled carefully. Thus the magnitudes were calculated using the most recent low temperature stellar atmosphere models of Hauschildt, Allard, \& Baron (1999) and folding them through the appropriate filter functions. The Cousins I \& R data were fitted simultaneously with the data in the HST filters.

\section{Discussion}

A closer inspection of the phased lightcurve shown in Figure 1 shows up two important features that were only hinted at by the ground based observations. Firstly, there is an asymmetry apparent in the lightcurve where the decrease in magnitude to minimum occurs more steeply than does the increase to maximum. The presence of excess emission after maximum means that the decrease to minimum begins at later phases than would be expected for a symmetric lightcurve. Secondly, the magnitude varies significantly between orbital phases 0.5 and 1.0 (see Figure 1) in successive orbits which is not seen at other orbital phases. This can be seen most clearly in Figure 2 where all 7 orbits obtained in the $\mathrm{f} 675 \mathrm{w}$ filter are shown. The greatest contrast (up to a magnitude) is seen between the observations indicated by the filled circles and the crosses.

Modelling of the lightcurve is of course limited by these complications, a simple synchronously rotating companion resulting in a symmetric lightcurve is no longer a complete model. If we fit the complete data set we find that we overestimate the flux in the rising part of the lightcurve. The resultant best-fit model is of a Roche lobe filling companion star which converts approximately $30 \%$ of the incident pulsar spin-down energy into optical flux.

Alternatively we can consider that the extra variability which is seen during the falling part of the lightcurve is associated with some other source of emission. Where we believe that the underlying system must be synchronously rotating and evenly heated (i.e. no hot spots) and thus has a symmetric lightcurve. Taking this assumption we fit just the rising part of the lightcurve. This results in a model with a much smaller companion star which less than half fills its Roche lobe and is somewhat more irradiated. This is similar to the original model discussed by Stappers et al (1999). It is important to note that the distance and extinction do play a strong role in determining the radius but the constraints are weak due to the quality of the fit.

Despite these limitations on the model fitting there are three parameters which are well constrained. The initially most surprising constraint is that the system is only moderately inclined, that is $i \sim 40$ degrees. However this small inclination adds support to the idea that the reason why the eclipses of PSR J2051-0827 have such a strong frequency dependence and show such variability is because the radio beam intercepts only the outer limits of the star or possibly 


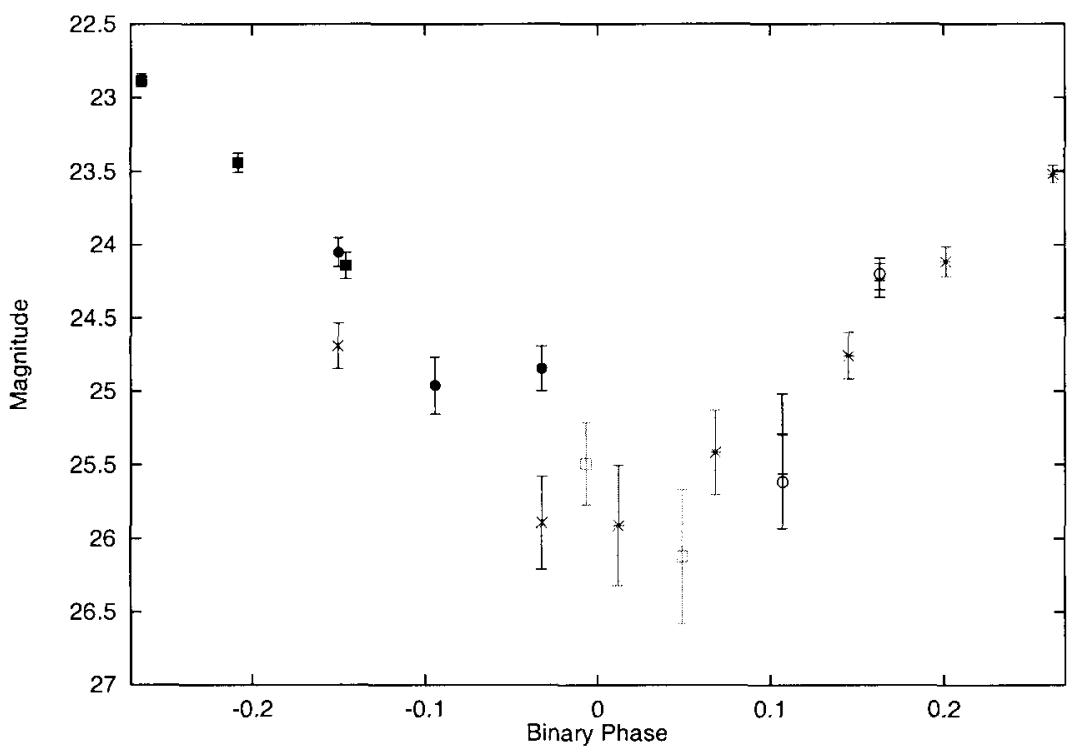

Figure 2. The magnitude of the companion in the HST filter $6675 \mathrm{w}$ during seven orbits of the system are shown. Observations from the same orbit are indicated by the same symbol.

just its wind. It is also apparent that a large fraction, greater than $30 \%$, of the pulsar's spin-down energy is converted in optical emission and the backside temperature of the star is very cool, likely less than $3000 \mathrm{~K}$.

Spectral observations and also photometry of a number of contiguous orbits is required to better understand the cause of the asymmetry and variability in the lightcurve. If some fraction of the emission that we see is coming from a shock above the companion's surface then that would clearly show up in a spectrum of the system. The timescale for the variability can only be determined by more intensive observing sessions than those possible with the observing constraints of the $H S T$.

\section{References}

Callanan, P. J., van Paradijs, J., \& Regelink, R. 1995, ApJ, 439, 938

Hauschildt, P. H., Allard, F., \& Baron, E. 1999, ApJ, 512, 377

Holtzman, J. A., et al. 1995, PASP, 107, 1065

Stappers, B. W., van Kerkwijk, M. H., Lane, B., \& Kulkarni, S. R. 1999, Ap.J, $510 \mathrm{~L}, 45$

Stappers, B. W., Bessell, M. S., \& Bailes, M. 1996a, ApJ, 473L, 119

Stappers, B. W., et al. 1996b, ApJ, 465L, 119 